\title{
Is Homocysteine an Independent Cardiovascular Risk Factor Also in Japanese?
} Key words: new risk factor, cardiovascular disease, atheroscle-
rosis, elderly

Homocysteine, a sulfur-containing amino acid, is produced solely during the metabolism of the essential amino acid methionine. Elevated plasma levels of total plasma homocysteine have been associated with increased risk of cardiovascular disease including both arterial atherosclerotic disease and venous thrombosis (1). Recent epidemiological evidence suggests that even mildly increased concentrations of homocysteine are associated with cardiovascular risk, and homocysteine has been recognized as a new independent cardiovascular risk factor. However, the majority of previous studies which showed a link between homocysteine and cardiovascular risk has been on Caucasian subjects. There is some recent evidence that it is also a risk factor in South Indian Asians (2) and other populations in Asia (3). The potential association between homocysteine and cardiovascular disease in Japanese subjects has not been well defined, although Japanese patients with cerebrovascular disease have higher homocysteine levels compared with control subjects (4).

In this issue, Kawamoto et al (5) reported that carotid atherosclerosis evaluated by ultrasonography has been associated with plasma homocysteine levels in the elderly Japanese subjects.

See also 1189.

In their study, even after controlling for age, body mass index, and various risk factors, high plasma homocysteine ( $>10$ $\mu \mathrm{mol} / l)$ was independently associated with both intima-medial thickness (Odds ratio $=6.5$ ) and carotid plaque (Odds ratio $=4.5$ ). In another recent case-control study, the age- and gender-adjusted odds ratio of cardiovascular disease was 6.5 in the highest plasma homocysteine quintile (mean: $30 \mu \mathrm{mol} / l$ ) when compared with the lowest quintile (mean: $5.9 \mu \mathrm{mol} / l)(6)$ ). These studies indicate that homocysteine is closely associated with clinical and subclinical atherosclerotic cardiovascular diseases independent of other cardiovascular risk factors also in a Japanese population. Among Japanese, the relative contribution of homocysteine to development of cardiovascular disease may be greater compared to Caucasians, due to the lower prevalence of hyperlipidemia.

The mechanism responsible for the association between hyperhomocysteinemia and cardiovascular disease remains unknown, but may be related to homocysteine-mediated endothelial cell injury, impaired endothelium-dependent vasodilation, increased oxidant stress, activation of platelets, and promotion of a thrombotic diathesis (7-12). It is well known that the association between hyperhomocysteinemia and cardiovascular disease is independent of other conventional risk factors. In addition this association was also independent of in vivo markers of thrombin generation (prothrombin fragment 1+2) and of tissue factor-induced coagulation activation (activated coagulation factor VII) $(6,13)$. Mild renal dysfunction is closely associated with both hyperhomocysteinemia and cardiovascular disease. However, the homocysteine level was associated with carotid atherosclerosis and clinical cardiovascular disease after controlling for serum creatinine and uric acid levels in Japanese $(5,6)$.

Plasma homocysteine concentrations are usually inversely related to plasma levels of folic acid and vitamins $B_{6}$ and $B_{12}$, which are cofactors or substrates for enzymes involved in homocysteine metabolism. The association between these vitamins and the plasma homocysteine level might be different between Asians and Caucasians. In previous studies of young and elderly Caucasians, the homocysteine levels were negatively correlated with plasma levels of vitamins $B_{6}$ and $B_{12}(14$, 15). In our study on elderly Japanese subjects, folic acid levels were negatively related to homocysteine levels (6), as previously shown in Caucasians $(14,15)$. In contrast, vitamin $B_{12}$ levels were unrelated to plasma homocysteine levels, despite the presence of subjects with relatively low levels of vitamin $B_{12}(6)$. In another study of Asian subjects, homocysteine levels were also unrelated to vitamin $B_{12}$ levels, but also were unrelated to plasma folic acid concentrations (7). It is uncertain whether the lack of association between homocysteine and vi$\operatorname{tamin} B_{12}$ is true for the general Japanese population or it is restricted to our study population. The validity of these results and the mechanisms responsible for this lack of effect remains unknown. International studies in a larger population are required to clarify these issues.

Recently, the alanine/valine (A/V) gene polymorphism of 5, 10-methylenetetrahydrofolate reductase (MTHFR), one of the key enzymes that catalyzes the remethylation of homocysteine, was reported. The VV genotype is correlated with increased plasma homocysteine levels as a result of the reduced activity and increased thermolability of this enzyme. Recent studies demonstrated that the VV genotype, which is commonly found in the Japanese population, was accompanied by in- 
creased plasma homocysteine levels. In addition, this genotype was associated with both ischemic stroke and coronary artery disease $(16,17)$. Recent review analysis disclosed that this genetic variation was associated with an increased risk for coronary artery disease in Japan, but not in other populations (18).

The definition of normal homocysteine level still remains controversial. Total plasma homocysteine levels ranging from 5 to $15 \mu \mathrm{mol} / l$ in healthy adults are used by many laboratories to define noralities, and levels exceeding this range have been referred to as mild $(15-30 \mu \mathrm{mol} / l)$, intermediate $(30-100 \mu \mathrm{mol} /$ $l$ ), and severe ( $>100 \mu \mathrm{mol} / l)$ hyperhomocysteinemia. However, this classification is arbitrary and plasma homocysteine levels increase along with aging and show gender difference (1-1.5 $\mu \mathrm{mol} / l$ lower in women). It is clear that the "normal range" still requires more precise definition.

Homocysteine levels may be lowered safely and effectively with diet modification and/or vitamin supplementation of folic acid, vitamin $B_{12}$, and $B_{6}$. Particularly, folic acid supplementation is effective (15). Folic acid supplements of $0.5-5 \mathrm{mg} /$ day reduce homocysteine levels $25-30 \%$ and a daily multivitamin containing at least $400 \mu \mathrm{g}$ of folic acid may have a similar effect. Thus, plasma homocysteine levels could be successfully reduced by folic acid therapy. As the next step, a randomized clinical study to demonstrate benefit on cardiovascular events is necessary.

In conclusion, in Japanese subjects as well as Caucasians, plasma homocysteine seems to be an independent risk factor for clinical and subclinical atherosclerotic cardiovascular disease. Thus, screening for and treating hyperhomocysteinemia should be proposed also for the Japanese patients, and those particularly with cardiovascular disease or advanced subclinical atherosclerosis.

Kazuomi KaRIO, MD, PhD, FACC, FACP The Department of Cardiology, Jichi Medical School, 3311-1 Yakushiji, Minamikawachi, Kawachi, Tochigi 329-0498

\section{References}

1) Duell PB, Malinow MR. Homocyst(e)ine: an important risk factor for atherosclerotic vascular disease. Curr Opin Lipidol 8: 28-34, 1997.

2) Chambers JC, Obeid OA, Ŕefsum H, et al. Plasma homocysteine concen- trations and risk of coronary heart disease in UK Indian Asian and European men. Lancet 355: 523-527, 2000

3) Lolin YI, Sanderson JE, Cheng SK, et al. Hyperhomocysteinaemia and premature coronary artery disease in the Chinese. Heart 76: 117-122, 1996.

4) Araki A, Sako Y, Fukushima Y, Matsumoto M, Asada T, Kita T. Plasma sulfhydryl-containing amino acids in patients with cerebral infarction and in hypertensive subjects. Atherosclerosis 79: 139-146, 1989.

5) Kawamoto R, Doi T, Tokunaga H. Association of plasma homocysteine concentrations with carotid atherosclerosis in elderly Japanese. Intern Med 40: 1189-1194, 2001

6) Kario K, Duell PB, M. Matsuo T, et al. High plasma homocyst(e)ine levels in elderly Japanese patients predict cardiovascular disease risk independently from markers of coagulation and endothelial cell damage. Atherosclerosis 157: 441-449, 2001.

7) Hajjar KA. homocyst(e)ine-induced modulation of tissue plasminogen activator binding to its endothelial cell membrane receptor. J Clin Invest 91: 2873-2879, 1993.

8) Nishinaga M, Ozawa T, Shimada K. Homocyst(e)ine, a thrombogenic agent, suppresses anticoagulant heparan sulfate expression in cultured porcine aortic endothelial cells. J Clin Invest 92: 1381-1386, 1993.

9) Stamler JS, Osborne JA, Jaraki O, et al. Adverse vascular effects of homocyst(e)ine are modulated by endothelium-derived relaxing factor and related oxides of nitrogen. J Clin Invest 91: 308-318, 1993.

10) Lentz SR, Sobey CG, Piegors DJ, et al. Vascular dysfunction in monkeys with diet-induced hyperhomocyst(e)inemia. J Clin Invest 98: 24-29, 1996.

11) Kokame K, Kato H, Miyata T. Homocyst(e)ine-respondent genes in vascular endothelial cells identified by differential display analysis. J Biol Chem 271: 29659-29665, 1996.

12) Lang D, Kredan MB, Moat SJ, et al. Homocysteine-induced inhibition of endothelium-dependent relaxation in rabbit aorta: role for superoxide anions. Arterioscler Thromb Vasc Biol 20: 422-427, 2000.

13) Kario K, Miyata T, Sakata T, Matsuo T, Kato H. Fluorogenic assay of activated factor VII: Plasma factor VIIa levels in relation to arterial cardiovascular diseases in Japanese. Arterioscler Thromb 14: 265-274, 1994.

14) Selhub J, Jacques PF, Wilson PWF, Rush D, Rosenberg IH. Vitamin status and intake as primary determinations of homocyst(e)inemia in an elderly population. JAMA 270: 2693-2698, 1993.

15) Malinow MR, Duell PB, Hess DL, et al. Reduction of plasma homocyst(e)ine levels by breakfast cereal fortified with folic acid in patients with coronary artery disease. N Engl J Med 338: 1009-1015, 1998.

16) Morita $H$, Taguchi J, Kurihara $H$, et al. Genetic polymorphism of 5, 10 methylenetetrahydrofolate reductase (MTHFR) as a risk factor for coronary artery disease. Circulation 95: 2032-2036, 1997.

17) Morita $H$, Kurihara $H$, Tsubaki $S$, et al. Methylenetetrahydrofolate reductase gene polymorphism and ischemic stroke in Japanese. Arterioscler Thromb Vasc Biol Sep 18 (9): 1465-1469, 1998.

18) Jee SH, Beaty TH, Suh I, Yoon Y, Appel LJ. The methylenetetrahydrofolate reductase gene is associated with increased cardiovascular risk in Japan, but not in other populations. Atherosclerosis 153: 161-168, 2000. 